\title{
Traumatismo vesical por arma de fuego: presentación de un caso y revisión de la literatura
}

\author{
Pérez Fentes DA, Toucedo Caamaño V, Villasenín Parrado L, Lema Grille J, Rodríguez Núñez H, \\ Blanco Parra M.
}

Servicio de Urología. Complejo Hospitalario Universitario de Santiago de Compostela. La Coruña

Actas Urol Esp. 2006;30(9):947-953

\section{RESUMEN}

\section{TRAUMATISMO VESICAL POR ARMA DE FUEGO: PRESENTACIÓN DE UN CASO Y REVISIÓN DE} LA LITERATURA

Objetivo: Presentar un caso de traumatismo vesical por arma de fuego y revisar aspectos diagnósticos y terapéuticos en la literatura relacionada.

Material y métodos: Presentamos el caso de un varón de 43 años con herida abdominal por arma de fuego y hematuria macroscópica. Diagnóstico de imagen mediante TC abdómino-pélvico y TC-cistografia. Tratamiento quirúrgico.

Resultados: Rotura vesical extraperitoneal en TC-cistografia. Laparotomía exploradora para descartar lesiones asociadas. Cierre quirúrgico de la pared vesical y ureteroneocistostomía con técnica transvesical por desinserción ureteral distal. Antibioterapia de amplio espectro. Evolución favorable. Alta al $14^{\circ}$ día.

Conclusiones: Los traumatismos vesicales por arma de fuego son una entidad poco frecuente. Debe realizarse exploración y cierre quirúrgico de la perforación vesical en todos los casos. En pacientes estables hemodinámicamente, TC abdómino-pélvico y TC-cistografía permiten descartar lesiones asociadas y clasificar el tipo de traumatismo vesical. Daño ureteral asociado en 5-8,9\%, diagnosticado intraoperatoriamente. Antibioterapia intravenosa de amplio espectro en todos los pacientes.

Palabras clave: Traumatismo vesical. Arma de fuego. TC-cistografia. Tratamiento.

\section{ABSTRACT \\ GUNSHOT BLADDER TRAUMA: CASE REPORT AND LITERATURE REVIEW.}

Objetive: To report one case of gunshot bladder trauma and to review its diagnosis and treatment in the related literature.

Methods: We report the case of a 43-year-old-male with an abdominal firearm wound and gross haematuria. Imaging diagnosis by abdominal and pelvic CT and CT-cystography. Surgical treatment.

Results: CT-cystography showed extraperitoneal bladder rupture. Exploratory laparotomy to evaluate for other associated injuries. Bladder wall surgical closure and ureteroneocystostomy with transvesical technique because of complete distal ureter tear. Broad spectrum antibiotherapy. Favourable progress. The patient was discharged at $14^{\text {th }}$ day.

Conclusions: Firearm bladder traumas are a rare entity. Surgical exploration and closure is mandatory. In haemodynamicaly stable patients, abdomen and pelvis CT and CT-cystography allow us to rule out associated injuries and to classify the bladder trauma type. Ureteral damage associated in 5$8,9 \%$, diagnosed during surgical exploration. Broad-spectrum antibiotherapy in all patients. 
$E_{\text {s }}$ lo era que don Victor Quintanar se arrastraba sobre la yerba cubierta de escarcha, y mordía la tierra. La bala de Mesía le había entrado en la vejiga, que estaba llena. Esto lo supieron poco después los médicos, en la casa nueva del Vivero, adonde se trasladó, como pudo, el cuerpo inerte del digno magistrado."

Así describe Leopoldo Alas "Clarín" en 1883 en su novela "La Regenta" una herida vesical por bala, destacando su mal pronóstico ${ }^{1}$. En aquel tiempo "dejar y ver" era la actitud predominante ante heridas abdominales penetrantes. Con el tiempo, la exploración quirúrgica precoz y los avances en técnica quirúrgica y anestesia producidos a raíz de las grandes guerras del siglo $\mathrm{XX}$ mejoraron el pronóstico de estos pacientes.

La medicina en general y el área quirúrgica urológica en concreto avanzaron de forma muy significativa a raíz de estas guerras. Bajo condiciones estresantes, sin la infraestructura adecuada y sin la experiencia requerida, cirujanos civiles y militares aprendieron cómo realizar drenajes urinarios con catéter uretral o cistostomía, cómo desbridar tejidos desvitalizados, las ventajas de retirar cuerpos extraños del aparato génito-urinario... ${ }^{2}$ En guerras más recientes, el menor tiempo en la evacuación del soldado herido desde la línea de fuego hasta el quirófano, se añade a los avances anteriores como factor de buen pronóstico ${ }^{3}$.

Las heridas por arma de fuego con implicación del aparato urinario son poco frecuentes en la población general y pocas veces publicadas. La intención de este artículo es presentar un caso de herida vesical por arma de fuego y realizar una revisión en la literatura internacional relacionada del diagnóstico y tratamiento de estas lesiones.

\section{CASO CLÍNICO}

Presentamos el caso de un varón de 43 años, sin alergias medicamentosas conocidas ni antecedentes médicos o quirúrgicos de interés, con herida abdominal por arma de fuego (pistola con proyectil $9 \mathrm{~mm}$ parabellum). El paciente ingresa estable hemodinámicamente en el servicio de Urgencias, con intenso dolor abdominal. En la exploración física se observa el orificio de entrada del proyectil en fosa iliaca izquierda con salida por glúteo izquierdo, con dolor abdominal y signos de irritación peritoneal principalmente en hipogastrio y fosa iliaca izquierda. No se aprecia afectación de genitales externos ni uretrorragia. Tras realizarse cateterismo uretro-vesical, se observa hematuria macroscópica intensa. En la analítica de ingreso destaca únicamente la presencia de anemia $(\mathrm{Hb}$ : 9,2 g/dl), siendo el resto de parámetros bioquímicos, de hemograma y coagulación normales.

Dada la estabilidad hemodinámica del paciente, se decide completar el estudio mediante pruebas de imagen previas a cirugía. Dado el teórico trayecto del proyectil (una vez conocidos los orificios de entrada y salida), con la sospecha de herida vesical por arma de fuego y dada la posible afectación de otros órganos, se decide realizar TC abdómino-pélvico y TC-cistografía (mediante replección vesical por gravedad con 300 cc de material de contraste diluido).

- TC abdómino-pélvico y TC-cistografia: rotura vesical póstero-lateral izquierda extraperitoneal, con extravasación de contraste tras el relleno. Mínima dilatación del sistema colector izquierdo. Herida de entrada en fosa iliaca izquierda con áreas de hemorragia en: mesosigma, cara lateral izquierda vesical, vesícula seminal izquierda y áreas perirrectal e isquiorrectal izquierdas. (Figs. 1 y 2).

Con estos hallazgos, se decide laparotomía exploradora. Con el paciente en posición de decúbito supino se realiza laparotomía media suprainfraumbilical. Inicialmente se procede a la reparación de una pequeña perforación en mesosigma sin afectación del asa sigmoidea y a la reparación de una mínima lesión a nivel de vena iliaca primitiva izquierda, comprobando posteriormente la indemnidad del resto del eje ilíaco arterial y venoso.

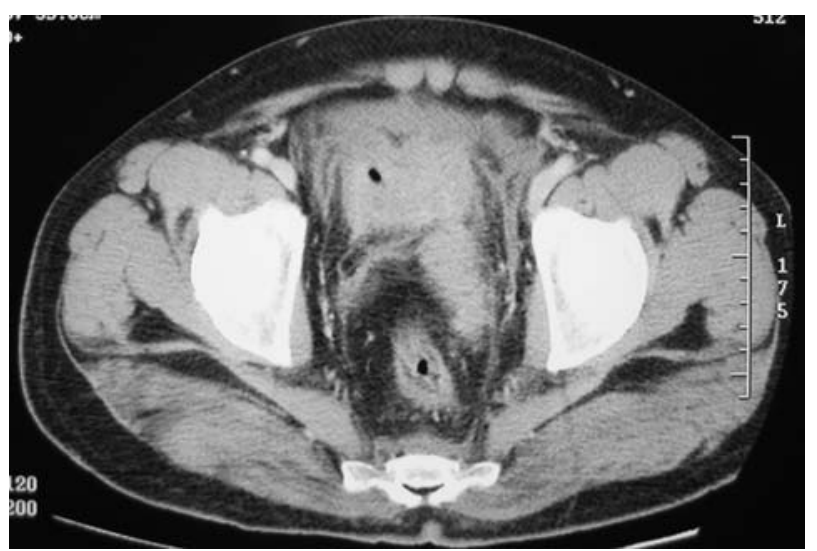

FIGURA 1. TC abdómino-pélvico. 


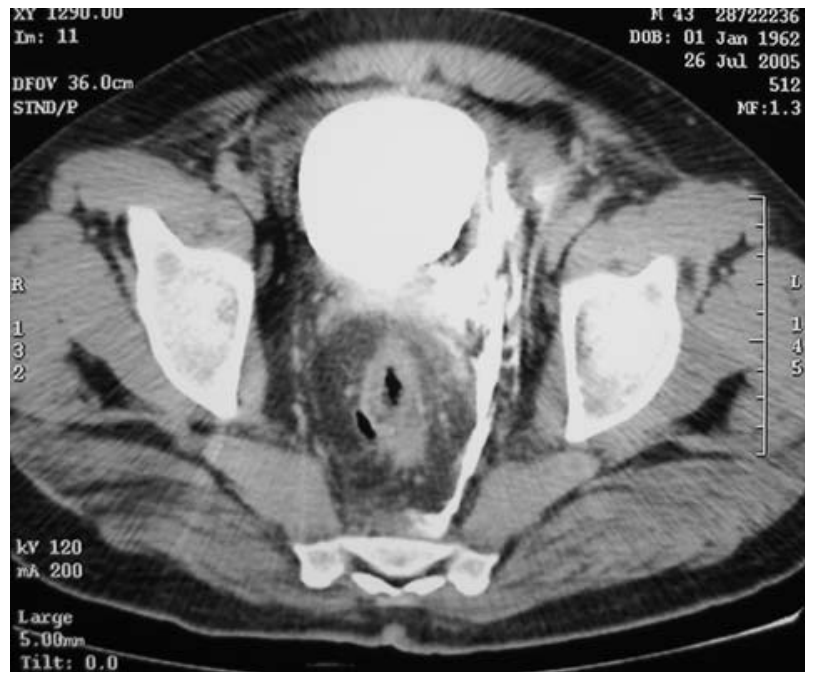

FIGURA 2. TC-cistografia: perforación vesical extraperitoneal.

A continuación se realiza cistotomía media longitudinal anterior y se observa una doble perforación vesical, correspondiente a los orificios de entrada (de $4 \mathrm{~cm}$ en fondo vesical) y salida (de 1$2 \mathrm{~cm}$, en región retromeática izquierda) del proyectil. Tras explorar la indemnidad del uréter distal izquierdo con catéter ureteral $5 \mathrm{~F}$, se comprueba que éste está desinsertado por completo en su porción intramural y que el proyectil en su recorrido también secciona el conducto deferente izquierdo. Se decide realizar sutura en dos planos de la doble perforación vesical, previo desbridamiento de los bordes desvitalizados y realizar reimplante ureteral con técnica transvesical y catéter doble $\mathrm{J}(26 \mathrm{~cm} / 6 \mathrm{~F})$ en pared posterior, previa liberación ureteral distal y sección de su extremo dañado. Tras realizar cierre, también en doble plano, de la cistotomía longitudinal anterior, se deja sonda uretro-vesical y doble drenaje cerrado de tipo aspirativo: uno a nivel intraperitoneal y el otro extraperitoneal, separado de la línea de sutura vesical.

Se pauta antibioterapia intravenosa de amplio espectro: Ceftriaxona 2 gramos cada 24 horas y Metronidazol 1,5 gramos cada 24 horas, transcurriendo el postoperatorio inicial de forma favorable. Al 5o día el paciente presenta deterioro del estado general, hipotensión, fiebre y leucocitosis con marcada desviación izquierda ( $31 \%$ cayados). Se sustituye la antibioterapia, de forma empírica, en espera del resultado de los hemocultivos por Imipenem 500 miligramos cada 6 horas intrave- noso, mejorando de forma significativa el paciente tanto clínica como analíticamente. En los hemocultivos crece un E.coli productor de b-lactamasas de espectro extendido, sensible a Imipenem. Se realiza un nuevo TC abdómino-pélvico que únicamente evidencia cambios post-quirúrgicos, descartándose la existencia de colecciones líquidas potencialmente causantes del cuadro séptico.

El resto del postoperatorio transcurre sin incidencias, retirándose inicialmente ambos drenajes y, al $10^{\circ}$ día, la sonda uretro-vesical. Finalmente se decide el alta del paciente tras completar 10 días de antibioterapia intravenosa. Se sustituye por Ciprofloxacino 250 miligramos cada 12 horas vía oral, según antibiograma previo, hasta la retirada del catéter JJ.

$\mathrm{Al}$ mes de la intervención el paciente acude de nuevo a nuestro servicio para realizar uretro-cistoscopia y retirada de catéter JJ. Se observa que la doble herida vesical está completamente cicatrizada, con la sutura del plano mucoso reabsorbida casi por completo (Fig. 3). El catéter JJ que tutorizaba la ureteroneocistostomía (Fig. 4) se retira endoscópicamente sin problemas. Actualmente, tres meses después de la intervención, el paciente está asintomático desde el punto de vista urológico.

\section{DISCUSIÓN}

La afectación genitourinaria representa menos del $1 \%$ del conjunto de las heridas por arma de fuego ${ }^{4}$ y es una entidad rara en el contexto de la población civil ${ }^{4,5}$.

Las características de las armas de fuego y sus proyectiles producen heridas en las que se suelen lesionar varios tejidos y órganos. Es importante descartar esas posibles lesiones asociadas ante una afectación genitourinaria por arma de fuego ${ }^{3-5}$.

Existen múltiples factores que determinan el gran poder lesivo de estas armas de fuego y que conviene tener en cuenta ${ }^{4,5}$ :

- Distancia a la que se efectúa el disparo: en general, los disparos a corta distancia producen heridas de entrada únicas con una gran destrucción tisular, los disparos a distancia intermedia presentan múltiples pequeñas heridas de menor capacidad destructiva y los disparos a larga distancia suelen tener escasa repercusión interna debido a la fragmentación del proyectil previa al impacto. 


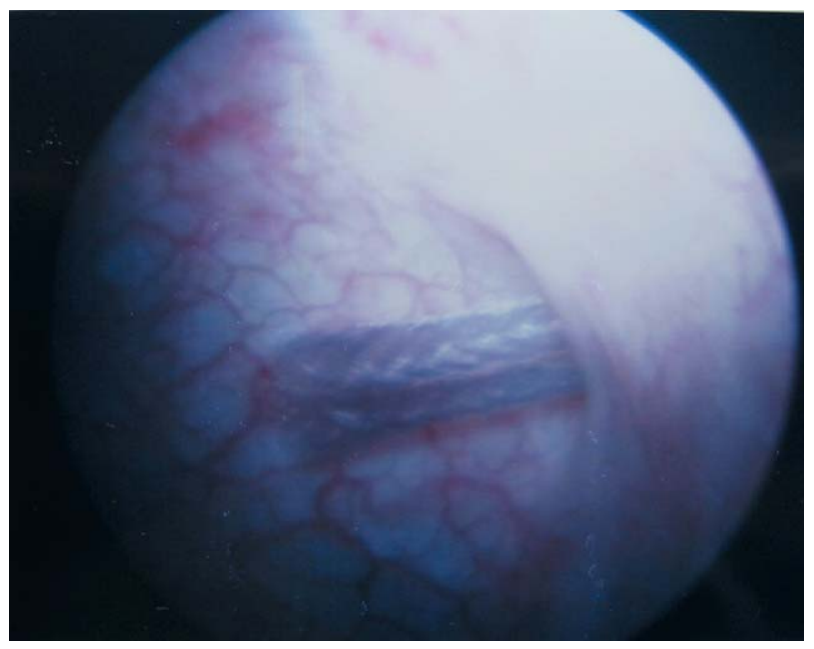

FIGURA 3. Sutura intravesical de herida vesical por arma de fuego: visión endoscópica.

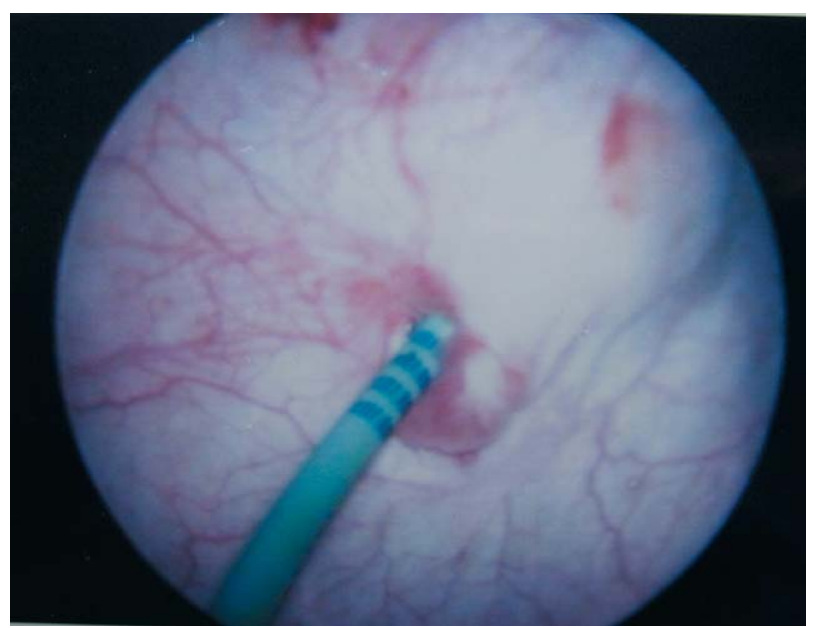

FIGURA 4. Catéter JJ tutorizado ureteroneocistostomia. Visión endoscópica.

- Energía cinética $\left(\mathrm{E}_{\mathrm{k}}\right)$ del proyectil empleado: $\left(\mathrm{E}_{\mathrm{k}}=1 / 2 \mathrm{x}\right.$ masa $\mathrm{x}$ velocidad $\left.{ }^{2}\right) . \mathrm{La}_{\mathrm{k}}$ del proyectil en el momento del impacto es directamente proporcional a la masa del mismo y al cuadrado de su velocidad y es transferida al objetivo por completo, causando la lesión.

- Forma del proyectil.

- Localización del orificio de entrada: en el abdomen, las lesiones son mayores que en la pelvis, debido a que no existe una estructura ósea protectora que disminuya la velocidad del proyectil $\mathrm{y}$, por tanto, $\mathrm{su}_{\mathrm{k}}{ }^{6}$.

- Trayectoria del proyectil: en la pelvis, las trayectorias de entrada posteriores o laterales son menos lesivas que las heridas frontales (que atra- viesan fácilmente el hipogastrio) o inferiores (que alteran el periné), donde los daños y las complicaciones son mayores ${ }^{6}$.

- Cavidad temporal: los proyectiles en el interior del organismo forman a su paso una gran cavidad temporal. Las lesiones tisulares están en función de la capacidad de cada órgano para amortiguar esta gran expansión: los órganos sólidos o los órganos en replección (por ejemplo: una vejiga distendida) la toleran peor que el intestino vacío o el músculo.

- Desaceleración: la desaceleración brutal del proyectil en el interior del organismo tiene un considerable poder lesivo.

Dentro de la afectación del aparato urinario bajo, la vejiga es el órgano más frecuentemente afectado $^{5}$ y suele asociarse a otras lesiones genitourinarias (uretra posterior, uréter...). Además, en un $76-95 \%$ presentan lesiones asociadas ${ }^{4}$ nogenitourinarias (intestino delgado, sigma, recto, tejidos blandos, estructuras vasculares, nerviosas, osteomusculares...).

El paciente herido por arma de fuego suele presentarse clínicamente con dolor abdominal y asociar síntomas en función de los distintos órganos y tejidos lesionados ${ }^{7}$. Las lesiones vesicales deben sospecharse principalmente ante la presencia de hematuria macroscópica y dolor abdominal. Otros posibles hallazgos son: incapacidad para orinar, aumento del tamaño de escroto, periné y muslos por extravasación de orina, irritación peritoneal en roturas vesicales intraperitoneales... Es importante en estos pacientes analizar la localización de los orificios de entrada y salida (si existe) que nos permiten reconstruir, al menos de forma teórica, el trayecto del proyectil y los potenciales órganos lesionados.

La actitud ante estos pacientes debe ser siempre la exploración quirúrgica ${ }^{4,5,7,8}$. En pacientes hemodinámicamente inestables ésta debe ser inmediata, sin realizar pruebas de imagen complementarias. En pacientes estables, se recomienda realizar TC abdómino-pélvico y si existe sospecha de perforación vesical, asociar TC-cistografia.

La TC-cistografía puede realizarse en lugar de la cistografía convencional (presenta una sensibi- 
lidad media del 95\% y una especificidad del 100\%) y está especialmente indicada en pacientes que van a ser sometidos a una TC por otras lesiones asociadas ${ }^{6,9,10}$. Para su realización se requiere cateterismo uretro-vesical, por lo que debe descartarse clinicamente la existencia de una lesión uretral y ante la sospecha clinica de la misma, realizar uretrografía retrógrada. Una vez cateterizada la vejiga, debe conseguirse una adecuada distensión vesical instilando por gravedad al menos 350 cc de contraste diluido (la mayoría de los falsos negativos de esta técnica se deben a una inadecuada distensión vesical, que hace pasar inadvertidas perforaciones mínimas). Al contrario que en la cistografia convencional, esta técnica presenta la ventaja de no necesitar placas post-miccionales ${ }^{6,9,10}$. Los hallazgos tomodensitométricos nos permiten clasificar las lesiones vesicales en varios tipos (Tabla 1) e individualizar su tratamiento (Tabla 2).

Las heridas vesicales por arma de fuego (por tanto, perforantes) requieren siempre laparotomía exploradora. Con el paciente colocado en decúbito supino para un abordaje abdómino-pélvico anterior (se reserva la posición de litotomía para un abordaje combinado abdómino-perineal), se corrigen inicialmente las lesiones asociadas potencialmente más graves (principalmente lesiones vasculares), para proceder posteriormente a la exploración vesical. Todo cuerpo extraño intravesical debe ser retirado para evitar complicaciones futuras. Suzuki ${ }^{11}$ publica el caso de un diver-

Tabla 1

Clasificación de los traumatismos vesicales.

\begin{tabular}{|c|c|c|c|}
\hline Tipo & Nombre & Definición. Mecanismo & $\begin{array}{l}\text { Hallazgos } \\
\text { TC-cistografia }\end{array}$ \\
\hline Tipo 1 & Contusión & Rotura incompleta de la mucosa vesical & Normal \\
\hline Tipo 2 & Rotura intraperitoneal & $\begin{array}{l}\text { Rotura hacia cavidad peritoneal, } \\
\text { típicamente en traumatismos contusos } \\
\text { sobre vejiga distendida, principalmente } \\
\text { en la cúpula }\end{array}$ & $\begin{array}{l}\text { Material de contraste entre las } \\
\text { asas de intestino y en fondos de } \\
\text { saco peritoneales }\end{array}$ \\
\hline Tipo 3 & Lesión intersticial & Laceración parcial con serosa intacta & $\begin{array}{l}\text { Material de contraste intramural } \\
\text { sin extravasación }\end{array}$ \\
\hline Tipo 4 & Rotura extraperitoneal & $\begin{array}{l}\text { La más frecuente. } \\
\text { Principalmente en traumatismos penetrantes. } \\
\text { En traumatismos contusos se debe a } \\
\text { laceraciones por fragmentos óseos }\end{array}$ & \\
\hline Tipo $4 \mathrm{a}$ & Simple & & $\begin{array}{l}\text { Material de contraste limitado al } \\
\text { espacio pélvico extraperitoneal }\end{array}$ \\
\hline Tipo $4 \mathrm{~b}$ & Compleja & & $\begin{array}{l}\text { Material de contraste hacia la } \\
\text { pared abdominal anterior, pene, } \\
\text { escroto, periné... }\end{array}$ \\
\hline Tipo 5 & Rotura combinada & Intra y extraperitoneal & $\begin{array}{l}\text { Características de ambos tipos } \\
\text { de rotura vesical }\end{array}$ \\
\hline
\end{tabular}

Sandler CM et al. Lower urinary tract trauma. World J Urol. 1998;16:69-75.

Tabla 2

Actitud terapéutica ante traumatismos vesicales.

\begin{tabular}{lll}
\hline Traumatismo contuso & Rotura extraperitoneal & $\begin{array}{l}\text { Antibioterapia y drenaje urinario } \\
\text { (Si rotura del cuello vesical o laparotomía exploradora por } \\
\text { otras lesiones, realizar cierre quirúrgico) } \\
\text { Antibioterapia y cierre quirúrgico }\end{array}$ \\
& Rotura intraperitoneal & \\
Traumatismo penetrante & Cualquier tipo de rotura & Cierre quirúrgico \\
\hline
\end{tabular}


tículo vesical de aparición tardía tras herida por bala. También se describen casos de proyectiles alojados dentro de vejiga ${ }^{12}$, algunos expulsados espontáneamente con la micción ${ }^{13-16}$ y otros retirados endoscópicamente ${ }^{17}$. Para evitar este tipo de complicaciones, se debe insistir en la búsqueda sistemática del proyectil cuando no existe orificio de salida en el paciente, primero radiológicamente y después intraoperatoriamente ${ }^{14}$.

Las modalidades técnicas son distintas en función del tipo de rotura y de su localización. Las roturas vesicales intraperitoneales (normalmente en cúpula vesical) requieren sutura vesical (previo desbridamiento de los bordes desvitalizados) y peritoneal. Las roturas extraperitoneales deben explorarse y suturarse intravesicalmente, previa cistotomía longitudinal anterior. Las roturas combinadas (intra y extraperitoneales) requieren ambos tipos de técnica. Cuando las heridas están próximas al trígono o hay sospecha de lesión ureteral, es conveniente realizar la sutura de la herida previo cateterismo ureteral retrógrado, para descartar su afectación ${ }^{4}$. En casos de vejigas totalmente destruidas, las opciones terapéuticas pasan por la realización de ureterostomías simples o ureterostomía trans-ileal, según la técnica de Bricker. En todos los casos debe dejarse un drenaje cerrado de tipo aspirativo en el espacio de Retzius, separado de la línea de sutura vesical y realizar cobertura antibiótica de amplio espectro. En estas modalidades técnicas existen algunos puntos de controversia:

- Tipo de sutura a realizar: las referencias bibliográficas relacionadas sostienen que la pared vesical debe ser suturada en dos planos, reservando la sutura en un plano con puntos sueltos cuando la herida es detectada tardíamente o está contaminada.

- Modo de derivación urinaria: quizá este es el punto más controvertido en la literatura. Lo que es indiscutible es que la orina debe ser drenada para favorecer la cicatrización de las heridas vesicales reparadas. La mayoría de los autores proponen realizar cistostomía fuera de la línea de sutura y exteriorizada a través de la piel por contraincisión ${ }^{3}$. Mianné ${ }^{4}$ mantiene que la sonda uretral podría reemplazar la cistostomía si la herida vesical es puntiforme, única y aislada. $\operatorname{Haas}^{8}$ reserva la cistostomía para casos de lesión uretral que contraindican el cateterismo uretro-vesical. Finalmente otro grupo de autores proponen combinar ambos tipos de derivación.

- Duración de la derivación urinaria: algunos autores $^{8}$ proponen 7-10 días y otros ${ }^{4,5}$ mantienen la derivación hasta 2-3 semanas. Se recomienda comprobar la estanqueidad vesical mediante cistografía previa a la retirada del catéter o catéteres en cuestión.

La lesión ureteral se encuentra asociada ${ }^{4}$ en un $5-8,9 \%$ a las heridas vesicales por arma de fuego, principalmente en su porción retrovesical e intramural. Hudolin ${ }^{3}$ en su artículo de revisión sobre heridas urogenitales sucedidas durante la guerra de Bosnia, explica que el uso de armas de alta $\mathrm{E}_{\mathrm{k}}$ aumenta el riesgo de daño ureteral hasta un $2-15 \%$ del total de heridas urogenitales. El método más efectivo para el diagnóstico de una lesión ureteral asociada es la exploración directa intraoperatoria ${ }^{18}$. En ocasiones la lesión ureteral se produce de forma iatrogénica durante la exploración o sutura de las heridas vesicales cuando éstas están próximas al trígono, de ahí la ventaja de tutorizar el uréter retrógradamente. Ante estas lesiones, la actitud quirúrgica pasa por la búsqueda de uréter sano que nos permita la realización de una ureteroneocistostomía, según la técnica que prefiera el cirujano. En estos casos, el tutor ureteral JJ permanecerá durante 2-4 semanas para prevenir el riesgo de fistulas y estenosis.

\section{CONCLUSIONES}

Los traumatismos vesicales por arma de fuego son una entidad poco frecuente en la población civil. En todos los casos es obligatorio realizar exploración quirúrgica y cierre de la herida vesical. En pacientes hemodinámicamente estables, la realización de TC abdómino-pélvico permite el diagnóstico de otras lesiones asociadas. La TCcistografia, correctamente realizada, permite el diagnóstico y la clasificación del tipo de rotura vesical, con implicaciones terapéuticas. La asociación de lesión ureteral se diagnostica principalmente durante la cirugia. En todos los pacientes es necesario instaurar antibioterapia de amplio espectro. 


\section{REFERENCIAS}

1. Fariña LA. Una herida de bala vesical en la novela "La Regenta" de Leopoldo Alas "Clarín”. Actas Urol Esp. 2000; 24(10):775-778.

2. Herr HW. Urological injuries in the civil war. J Urol. 2004; 172:1800-1804.

3. HudolinT, Hudolin I. Surgical management of urogenital injuries at a war hospital in Bosnia-Herzegovina, 1992 to 1995. J Urol. 2003;169(4):1357-1359.

4. Mianne D, Guillotreau J, Lonjon T, Dumurgier C, Argeme M. Les plaies par arme à feu du bas appareil urinaire chez 1'homme. J Chir. 1997;134(4):139-153.

5. Tiguert R, Harb JF, Hurley PM, Gomes De Oliveira J, Castilla Frontera RJ, Triest JA et al. Management of shotgun injuries to the pelvis and lower genitourinary system. Urology 2000;55(2):193-197.

6. Salzano A, Nocera V, De Rosa A, Rossi, Ciamillo A, De Nisi R et al. Segni con Tomografia Computerizzata nel coinvolgimento Della pelvi nelle ferite da arma du fuoco. Esperienza personale. Radiol Med. (Torino) 2000;100(5):310-313.

7. Lynch D, Martínez-Piñeiro L, Plas E, Serafetinidis E, Turkeri L, Hohenfelner M. Guidelines on urological trauma. Update February 2003. Disponible en http://www.uroweb.org.

8. Haas CA. Ruptured bladder. En Resnick M. Critical decisions in urology, third edition. London, BC Decker Inc. 2004, pp 148-151.

9. Sandler CM, Goldman SM, Kawashima A. Lower urinary tract trauma. World J Urol. 1998;16(1):69-75.

10. Vaccaro JP, Brody JM. CT cystography in the evaluation of major bladder trauma. Radiographics 2000;20(5):1373-1381.

11. Suzuki K, Tanaka O, Saito T, Tokue A. Giant bladder diverticulum due to previous bullet injury: findings of gadolinium-enhanced magnetic resonance imaging. Int J Urol. 2002;9(9):517-519.
12. Halkic N, Wisard M, Abdelmoumene A, Vuilleumier H. A large bullet in the bladder. Swiss Surg. 2001;7(3):139140 .

13. Abdelsayed MA, Bissada NK, Finkbeiner AE, Redman JF. Spontaneous passage of bullet during voiding. South Med J. 1978;71(1):83-84.

14. Kilic D, Kilinc F, Ezer A, Guvel S. Spontaneous expulsion of a bullet via the urethra. Int J Urol. 2004;11(7):576-577.

15. Sankari BR, Parra RO. Spontaneous voiding of a bullet after a gunshot wound to the bladder: case report. J Trauma 1993;35(5):813-814.

16. DiDomenico D, Guinan P, Sharifi R. Spontaneous expulsion of an intravesical bullet. J Am Ostheopath Assoc. 1997;97(7):415-416.

17. Gulanikar A, Pandey P, Terrell F. A bullet in the bladder. Br J Urol. 1998;82(2):304.

18. Best CD, Petrone P, Demiray S, Kuncir E, Kimbrell B, Asensio JA. Traumatic ureteral injuries: a single institution experience validating the American Association for the Surgery of Trauma-Organ Injury Scale grading scale. J Urol. 2005;173(4):1202-1205.

Dr. D.A. Pérez Fentes

Servicio de Urología

Hospital Clínico Univ. de Santiago de Compostela Travesía Choupana s/n. 15706 Santiago de Compostela (La Coruña) E- Mail: danielfentes@yahoo.es

(Trabajo recibido el 7 de octubre de 2005) 